\title{
Response of Vessels to Ischaemia
}

\author{
G. A. GRESHAM \\ Cambridge
}

Ischaemia literally means the reduction or arrest of the blood supply to a tissue. The result is a state of hypoxia in the territory supplied by the compromised blood vessel. The effects of hypoxia on blood vessels in the pulmonary circulation have received considerable attention in the past but the effects on blood vessels in other parts of the body have not been so intensively studied. ${ }^{1}$ Arteries, veins and capillaries do not respond in the same way to hypoxia and arteries in some organs, such as the lung, respond differently to arteries in other organs such as the heart. In the eye, arteries and veins respond differently. In the hypoxic retina produced by simulated high altitude conditions small arteries dilate, larger ones constrict and veins are unaffected. Furthermore, the vascular response in the retina varies from one subject to another. ${ }^{2}$

To some extent the effects of ischaemia on blood vessels depends upon their structure. The central retinal artery has features similar to those of arteries in skeletal muscle. After the first division of the retinal artery the internal elastic lamina is lost. This affects the permeability of the vessel wall to substances such as lipid. ${ }^{3}$ The capillaries of the choroid have highly fenestrated endothelial cells again affecting vascular permeability. ${ }^{4}$

Ischaemia can be caused either by organic disease of blood vessels such as atherosclerosis or thrombosis or by functional changes such as vascular spasm or impaired blood flow due to changes in blood pressure or blood viscosity. The obvious effect of ischaemia is to produce defective oxygenation of the tissue.

In addition a variety of metabolic products accumulate which are numerous and have diverse effects on the vascular wall. Hypoxia may result from causes other than vascular obstruction and in general the effects upon the organ are similar to those produced by ischaemia. Pulmonary disease, circulatory shunts, anaemia and histotoxic agents can all lead to hypoxia.

In some animals and particularly in man arteries are naturally hypoxic. This is largely determined by the thickness of the vessel wall and the number of lamellar units composed of elastin and smooth muscle that are present. In thick walled vessels the hypoxic effects depends upon the adequacy of blood supply to the vessel by the vasa vasorum. In the dog vasa permeate the vessel wall as they do in veins in many species. In man vasa supply a limited territory of the outer third of the artery. Spontaneously occurring atherosclerosis in the dog is not described and extreme dietary and other measures are needed to produce it experimentally.

The inner part of the media of human arteries is a vulnerable structure. It survives on diffusion from the capillary bed of the vasa and upon diffusion of nutrients from the lumen of the artery. It is a precarious existence and any limitation of diffusion of nutrients as for example by intimal thickening leads to smooth muscle damage of the inner media. This is followed by a repair process which consists of smooth muscle cell proliferation. This, in turn, further thickens the arterial wall and aggravates the hypoxic state. Medial smooth muscle cells that have proliferated migrate to 
the intima and an atherosclerotic plaque is formed.

Intimal changes leading to atherosclerosis are the result of endothelial injury. The injury is hypoxic. Many agents damage endothelium but haemodynamic changes figure largely in the process. Increased intraluminal pressure leads to increased oxygen demand by the vessel wall. Rapid changes of pressure, either increase or decrease, reduce flow in the vasa vasorum producing hypoxic conditions. Rapid flow can produce axial streamlining effects of red blood cells with flow stagnation. The localisation of atherosclerotic lesions is best explained by haemodynamic forces. ${ }^{6}$ Other factors contribute to the process, diabetes: cigarette smoking and hyperlipidaemia are examples. High low density lipoproteins (LDL) lead to transcellular and pinocytotic uptake by endothelial cells. Oxidisation of imbibed LDL gives rise to histotoxic substances which aggravate the atherosclerotic process.

Hypoxia leads to endothelial damage in a variety of ways. Enzyme activity is inhibited with a consequent fall in ATP reserves. This leads to impairment of sodium pump activity and consequent swelling of endothelial cells. More remote effects of hypoxia are on the renin-angiotensin mechanism leading to hypertension which, as we have seen, has deleterious effects on endothelial cells.

Even after the insult leading to hypoxia is reversed problems remain. Reperfusion of previously obstructed vessels leads to the production of free radical products which are cytotoxic. Another problem arises from the stimulation of previously damaged endothelial cells leading to new vessel formation. Experimentally induced arterial constriction in the dog leads to endothelial damage followed by endothelial cell regeneration as shown by labelling with tritiated thymidine. ${ }^{7}$ Mitoses occur subsequently in smooth muscle cells and adventitial fibroblasts. A variety of growth factors derived from platelets, macrophages and fibroblasts produces this effect. ${ }^{8}$

Neovascularisation after hypoxia is an important problem particularly in the eye.
The effects of drugs and vasoactive agents on the process are being studied. Using subcutaneous implants of plastic sponges in the rate of growth of new vessels in the sponges is being investigated. Vascular development is measured by the rate of radioactive xenon clearance instilled into the centre of the sponge and by morphometry using Ulex europeaus labelling of endothelial cells in histological sections. This is a useful model for the study of promoters and inhibitors of vascular proliferation. ${ }^{9}$

Ischaemic damage is a common pathological process which plays a part in many diseases of degenerative, inflammatory and neoplastic origins. The facts that come into play when ischaemia develops are numerous and as yet only partly elucidated. Changes in the walls of blood vessels made ischaemic are still controversial.

\section{Bibliography}

${ }^{1}$ Duke HN: Observations on the Effects of Hypoxia on the Pulmonary Vascular Bed. J Physiol 1957; 135: 45-9,

${ }^{2}$ Brinchmann-Hansen O and Myhre K. February. Vascular Response of Retinal Arteries and Veins to Acute Hypoxia at $8,000, .10,000,12,500$ and 15,000 Feet of Simulated Altitude. Aviation, Space and Environmental Medicine 1990; $112-116$.

${ }^{3}$ Noma A, Takahashi T and Wada T. Elastin Lipid Interaction in the Arterial Wall. Atherosclerosis 1981; 38: 373-6.

${ }^{+}$Pedro R and Marano C. Chorioretinal Ischaemia. Medicographia, 1988; 10: 10-13.

${ }^{5}$ Gresham GA. Early Events in Atherogenesis. Lancet 1975; i: 614-17

${ }^{6}$ Boxen I. Endothelial Hypoxia Induction of Atherosclerosis: An Explanation of Patency Rates for Internal Mammary Artery and Other Coronary Artery Bypass Grafts. Medical Hyotheses 1989; 30: $157-65$

${ }^{7}$ Pasyk S, Schaper W, Schaper J, Pasyk K, Miskiewicz G, Steinseifer B. D.N.A. Synthesis in Coronary Collaterals After Coronary Artery Occlusion in the Conscious Dog. Am J Physiol 1982; 242: 1031-7

${ }^{8}$ Schaper W, Hari S, Sharma MD, Quinkler W, Markert T, Wunsch M, Schaper J. Molecular Biological Concepts of Coronary Anastomoses. JACC 1990; 15: 513-18

${ }^{9}$ Andrade SP, Fan TPD, Lewis GP.Quantatitative in vivo Studies on Angiogenesis in a Rat Sponge Model. Br J Exp Pathol 1987; 68: 755-8 